\title{
Tensiones entre el pensamiento Kohutiano y Kernberiano; aportes para el enfoque relacional ${ }^{1}$
}

\author{
Adrián Ortiz Avila ${ }^{2}$ y Martha Sánchez Muñoz ${ }^{3}$ \\ CENTRO DE EDUCACIÓN E INVESTIGACIÓN EN PSICOANÁLISIS (CEIP), San Pedro Garza García, México
}

\section{Introducción.}

Me llamó mucho la atención el énfasis que Kohut hace sobre la empatía, la actitud del terapeuta más hacia la comprensión del paciente. Que menciona los déficits ambientales que tuvo el paciente en la historia de su desarrollo, y cómo esto hace que culmine en patologías de su self, y eso, en general y muy a grandes rasgos, sería lo que enferme a las personas, y por ello es a la vez una oportunidad en el espacio psicoanalítico para crear un mejor ambiente, más empático y cohesivo, que pueda ayudar al paciente.

A mediados de la década de 1960 en el campo psicoanalítico de Estados Unidos y en otras partes del mundo, se estaba dando una crisis en el teorizar y la técnica psicoanalítica, debido a que los pacientes que entraban en análisis no solo encajaban en los modelos del psicoanálisis tradicional; no solo se trataban de psicosis o neurosis transferenciales. Mucho del pesar de los pacientes eran patologías que tenían que ver con la forma de relacionarse con las demás personas.

Es aquí donde Otto Kernberg hace su aparición con sus primeros estudios sobre las "condiciones bordeline"

Kohut, por su parte, menciona que existen pacientes analizables, no psicóticos, no bordeline, pero que presentan patologías derivadas de su self, con un self grandioso.

\footnotetext{
${ }^{1}$ Ortiz Ávila, A. y Sánchez Muñoz, M. (2020). Tensiones entre el pensamiento Kohutiano y Kernberiano; aportes para el enfoque relacional. Clínica e Investigación Relacional, 14 (1): 244-247. [ISSN 1988-2939] [Recuperado de www.ceir.info ] DOI: 10.21110/19882939.2020.140115

${ }^{2}$ Médico-psiquiatra por la Universidad de Monterrey (UDEM), candidato en entrenamiento en psicoanálisis por el Centro de Educación e Investigación en Psicoanálisis (CEIP). Actualmente Secretario del Colegio de Psiquiatría y Psicofarmacología de Nuevo León A.C.

3 Psicóloga, clínica, Psicoanalista. 30 años de experiencia en la Consulta Privada atendiendo casos de psicoterapia y psicoanálisis. Directora de Instituto de Psicoanálisis del CEIPMty 2016-2018. EX- Directora Académica del Instituto de Psicoterapia A.B.P. Y EX- Directora de su Clínica en Monterrey, N.L. EXCoordinadora de la Clínica de la Asociación Regiomontana de Psicoanálisis A.C. Profesora y Supervisora invitada en la Maestría de Clínica Psicoanalítica de la Facultad de Psicología de la U.A.N.L. Líneas de investigación: psicoanálisis y género.
}

CeIR Vol. 14 (1) -Abril 2020 ISSN 1988-2939 - www.ceir.info

(c) Derechos reservados/Copyright de Clínica e investigación Relacional y los autores. Prohibida la reproducción total o parcial sin autorización expresa. Este material es para uso científico y profesional exclusivamente y puede contener información clínica sensible. Los editores no se responsabilizan de los contenidos de los autores. Dirigir las consultas sobre derechos y autorizaciones a ceir@psicoterapiarelacional.es 
Y me llama la atención las dos formas de pensar, tan diferentes de Kernberg y Kohut, y por ende, de entender la naturaleza humana, la naturaleza de la enfermedad mental, de la psicopatología en general. Porque vienen de dos enfoques muy distintos. Aunque ambos, tanto Kohut como Kernberg, son muy respetosos de los autores clásicos del psicoanálisis y no se despegan de la ciencia psicoanalítica pura, tienen los pies bien pegados a la tierra. Ambos fueron estudiosos de Freud, Melanie Klein, Mahler. Por ejemplo, al principio de sus estudios Kohut seguía en parte a Mahler, con la concepción de que el individuo nace indiferente al medio, en el estadio de la fase autista. Sin embargo, posteriormente Kohut replantea el problema desde el desarrollo, y considera que la persona nace con un self con capacidad para vincularse a los demás, y de hecho de ahí provienen las patologías mentales derivadas de la interacción del self con sus self objects.

Diferente a como lo plantea Kernberg, en donde él propone que la psique del individuo nace con carga libidinal y agresiva, ya como algo constitucional. Siguiendo muy de la mano a Klein, pero también a Freud. Continua con el tema de conflicto intrapsíquico, el papel de la fantasía, del mundo interno, muy relacionado con la escuela de relaciones objetales europea.

Debido a que los dos plantean un origen de la psicopatología diferente, esto derivará a un abordaje clínico diferente a los pacientes, una manera de diagnosticar, tratar y el sentido pronóstico. Por ello creo que es pertinente realizar la discusión, ya que es un tema de discusión en nuestros tiempos modernos. $Y$ son dos escuelas que han aportado mucho al psicoanálisis, no solo al psicoanálisis, sino a la psicoterapia, las terapias breves también, y a la generación de conocimiento psicoanalítico.

\section{Heinz Kohut y el desarrollo del self.}

Kohut definió el self como el núcleo de la personalidad, un contenido de aparato psíquico que forma parte tanto del yo como del ello y del superyó. Un self bien cohesivo seria la condición propicia para la salud mental; mientras que un self poco cohesivo seria la causa de mecanismos de defensa compensatorios, trastornos de personalidad y de enfermedad mental.

Según Kohut, el self y su cohesion se desarrollan y se construyen gracias a las relaciones narcisistas con los objetos arcaicos e infantiles.

Según Kohut, el self y su cohesión se desarrollan y se construyen gracias a las relaciones narcisistas con los objetos arcaicos e infantiles (objetos del self) por la interiorización 
transmutadora de esos objetos y de sus funciones que inicialmente espejan con empatía la grandiosidad del infante (self grandioso), se dejan idealizar (imago parental idealizado) y permiten vivencias gemelares de alter-ego. Para Kohut, un objeto de self es un objeto narcisista, es decir que está catectizado por la libido narcisista, hace parte del bebé y del infante.

Kohut llega a considerar la matriz relacional, en la que todo individuo se desempeña desde su nacimiento, como parte clave y en la cual se produce la psicopatología del individuo.

\section{Otto Kernberg, teoría de relaciones de objeto.}

Kernberg considera que la mente humana se estructura a partir de la internalización de las relaciones con los objetos importantes. El mundo interno de la persona comienza por introyectar los objetos de manera parcial (todo bueno o todo malo), asi como el estado afectivo que caracteriza esa relación y un componente cognitivo. La relación de papeles recíprocos entre el mundo interno del paciente y los objetos se expresa en fantasías y deseos.

\section{Puntos de discusión.}

1) El primer punto seria, de donde proviene cual es el modelo de la estructura de la mente de cada Teoría:

a. La lucha eterna entre si la persona tiene alguna carga de agresividad o la agresividad proviene de su relación con el ambiente.

2) Otro punto importante, la forma que conciben cada uno el narcisismo, que es de hecho uno de los grandes problemas al leer a Kernberg y compararlo con Kohut, y las formas de aplicar tratamientos, diagnósticos y pronósticos.

3) También en cuanto a la técnica. Si para Kohut el ambiente o las carencias de este es lo que daña al individuo y se desarrolla patología, es precisamente el nuevo ambiente psicoanalítico, con la empatía, el espejeo del analista el cual puede curar al paciente. En tanto, para Kernberg sería el lograr, primero entender al paciente, entenderlo el terapeuta en cuanto a sus mecanismos defensivos, y poder confrontarlo con su realidad.

Creo que el trabajo de pacientes narcisistas y con patología limítrofe ha dado mucho fruto en el campo del psicoanálisis, tanto en lo teórico como en lo clínico. Y ha dado pie 
a puntos de discusión muy importantes, como las dos maneras de teorizar entre Heinz Kohut y Otto Kernberg.

Tanto en el desarrollo de la teoría de Kernberg y la de Kohut se observa un respeto por los aportes de los primeros investigadores en psicoanálisis, por ejemplo, en su libro de Analisis del Self, Kohut menciona muchos conceptos freudianos, como catecsias, libido, que muchos autores modernos casi no manejan. Siento que guarda un gran respeto por los autores clásicos, sin dejar de dar su aportación.

En el desarrollo de la teoría de Kernberg, siempre se observa la presencia de M. Klein en sus aportes, ya que es eminentemente derivado de la teoría de relaciones de objeto.

\section{Referencias:}

Kernberg, Otto. (1993). La Teoría de las Relaciones Objetales y el Psicoanálisis Clínico. México: Paidos.

Kohut, Heinz. (1971). Análisis del Self. Argentina, Buenos Aires: Amorrortu.

Serra Undurraga Jacqueline, K. (2016). El diagnóstico del narcisismo: una lectura relacional. Rev. Asoc. Esp. Neuropsiquiatría, 1, 171 - 187.

Yildiz, Ismail. (2008). TEORÍAS SOBRE AFECTOS Y SÍNTOMAS III Psicología psicoanalítica del self (H. Kohut) y aportes de Otto Kernberg. Revista de la Asociación Psicoanalítica Colombiana, 1, 101 - 124. 